\title{
The design and characterization of a 300 channel, optimized full-band millimeter filterbank for science with SuperSpec
}

J. Redford, J. Wheeler, K. Karkare, S. HaileyDunsheath, C. M. Bradford, et al.

J. Redford, J. Wheeler, K. Karkare, S. Hailey-Dunsheath, C. M. Bradford, E. Shirokoff, P. S. Barry, G. Che, J. Glenn, H. G. Leduc, P. Mauskopf, R. McGeehan, T. Reck, J. Zmuidzinas, "The design and characterization of a 300 channel, optimized full-band millimeter filterbank for science with SuperSpec," Proc. SPIE 10708, Millimeter, Submillimeter, and Far-Infrared Detectors and Instrumentation for Astronomy IX, 1070810 (9 July 2018); doi: 10.1117/12.2313666

Event: SPIE Astronomical Telescopes + Instrumentation, 2018, Austin, Texas, United States 


\title{
The Design and Characterization of a 300 channel, optimized full-band Millimeter Filterbank for Science with SuperSpec
}

\author{
J. Redford ${ }^{\mathrm{a}}$, J. Wheeler ${ }^{\mathrm{b}}$, K. Karkare ${ }^{\mathrm{d}}$, S. Hailey-Dunsheath ${ }^{\mathrm{a}}$, C. M. Bradford ${ }^{\mathrm{a}, \mathrm{c}}$, E. Shirokoff ${ }^{\mathrm{d}}$, \\ P. S. Barry ${ }^{\mathrm{d}}$, G. Che ${ }^{\mathrm{e}}$, J. Glenn ${ }^{\mathrm{b}}$, H. G. Leduc ${ }^{\mathrm{c}}$, P. Mauskopf ${ }^{\mathrm{e}}$, R. McGeehan ${ }^{\mathrm{d}}$, T. Reck ${ }^{\mathrm{c}}$, and \\ J. Zmuidzinas ${ }^{\mathrm{a}, \mathrm{c}}$ \\ ${ }^{a}$ California Institute of Technology, 1200 E. California St. Pasadena, CA, 91125 USA \\ ${ }^{\mathrm{b}}$ Center for Astrophysics and Space Astronomy, University of Colorado Boulder, 2000 \\ Colorado Ave., Boulder, CO 80309, USA \\ ${ }^{\mathrm{c} J e t}$ Propulsion Laboratory, 4800 Oak Grove Dr., Pasadena, CA 91109, USA \\ ${ }^{\mathrm{d}}$ Department of Astronomy \& Astrophysics, University of Chicago, 5640 South Ellis Ave., \\ Chicago, IL 60637, USA \\ eSchool of Earth and Space Exploration and Department of Physics, Arizona State University, \\ Tempe, AZ 85287, USA
}

\begin{abstract}
SuperSpec is an integrated, on-chip spectrometer for millimeter and sub-millimeter astronomy. We report the approach, design optimization, and partial characterization of a 300 channel filterbank covering the 185 to 315 $\mathrm{GHz}$ frequency band that targets a resolving power $\mathcal{R} \sim 310$, and fits on a $3.5 \times 5.5 \mathrm{~cm}$ chip. SuperSpec uses a lens and broadband antenna to couple radiation into a niobium microstrip that feeds a bank of niobium microstrip half-wave resonators for frequency selectivity. Each half-wave resonator is coupled to the inductor of a titanium nitride lumped-element kinetic inductance detector (LEKID) that detects the incident radiation. The device was designed for use in a demonstration instrument at the Large Millimeter Telescope (LMT).
\end{abstract}

Keywords: Spectroscopy, Kinetic Inductance Detectors, Millimeter-Wave

\section{INTRODUCTION}

The far-infrared (FIR) contains at least half of the electromagnetic energy ever produced in stars and galaxies, and appears to have been the dominant emission pathway for the most active star-forming stages in galaxies. Understanding this dust-obscured star formation requires spectroscopic study at FIR / submillimeter and millimeter wavelengths; this band is both immune to dust obscuration so probes galaxies' bulk properties, and also offers a range of diagnostics of all interstellar medium phases: ionized, neutral, and molecular. One particular spectral tool useful for understanding star formation in the early universe is the $157.7 \mu \mathrm{m}$ fine-structure transition of ionizied carbon, [CII]. [CII] is among the most energetically important coolants of gas in galaxies across all wavelengths, and has been found to be a reasonable proxy for total star formation activity. ${ }^{1}$

[CII] is being pursued and detected in individual galaxies with ALMA in small numbers, but future instruments on single-dish telescopes can offer two classes of complementary approaches. A wideband, multi-object spectrometer (MOS) with on order 100 steered feeds on a 30-50 meter telescope ${ }^{2}$ can exceed the full-band galaxyby-galaxy spectral survey speed of ALMA by nearly an order of magnitude, yielding samples of tens of thousands of full band spectra of galaxies at all epochs. Alternatively, tomographic intensity mapping targeting [CII] at redshifts of $\sim 4$ to 9 offers the potential to measure the 3 -D clustering signal produced by [CII] in galaxies. The linear clustering signal on $\sim 0.1$ to 1 degree ( 10 to 100 comoving Mpc) scales measures the total [CII] luminosity, and thus potentially the total star formation, across all galaxies. ${ }^{3,4}$ This intensity mapping approach is particularly compelling for the reionization epoch as the steep faint-end slopes measured in the UV suggest that much of the emission may be originating in galaxies below the detection limit of ALMA or JWST. ${ }^{5,6}$

\footnotetext{
Send correspondence to E-mail: jredford@caltech.edu
} 
SuperSpec is designed to enable these new classes of instrument. It is a compact, wideband, on-chip spectrometer which is reduced to a single 'pixel.' Many SuperSpec spectrometers can populate a focal plane; and it can be the building block of a large MOS. This leans heavily on the inherent multiplexibility of the kinetic inductance detectors (KIDs), making them the natural detector choice for this goal of high-pixel-count MOS instruments. SuperSpec is implemented by having incident radiation coupled into a feedline and propagate through a bank of $\lambda / 2$ resonator filters, where $\lambda$ is the central wavelength of the channel, which couple the radiation onto a KID for readout. Our current design has a filterbank with 300 channels, spanning the frequency range of 185 to 315 $\mathrm{GHz}$ (the $1 \mathrm{~mm}$ atmosphere window ${ }^{7}$ ). Since even an optimally coupled resonator can only absorb $50 \%$ of the incident power, the resonators are placed a quarter-wave apart. This causes their reflections to be out of phase and cancel, meaning that more power will end up in one of the channels. This architecture is illustrated in a circuit diagram shown in fig. 1. This paper describes the design of the full 300 channel device intended to deploy at the Large Millimeter Telescope (LMT) in early 2019, for an on-sky demonstration.

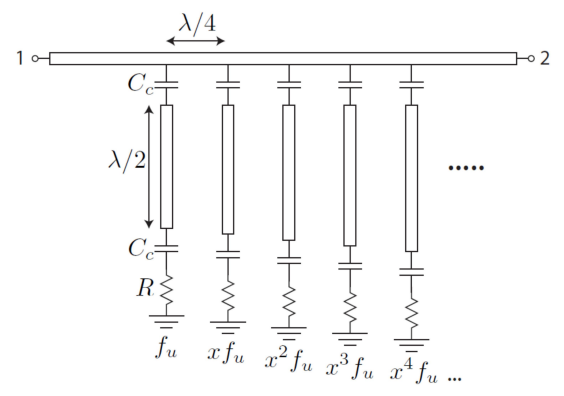

Figure 1. Shown is a circuit diagram of the SuperSpec architecture. The radiation coupled in from free space propagates down the feedline from port 1 to port 2. A bank of half-wave resonators is used to filter out specific frequency bands and couple the power to absorbing KID detectors, shown as resistors in the diagram. The resonators are placed a quarter wavelength apart so that their reflections will destructively interfere (like an anti-reflective coating in optics). Figure from ref 8 .

\section{DEVICE DESIGN}

The basic filterbank approach employed by SuperSpec is shown in fig. 1. The optimization of the filterbank involves several parameters. Since the filterbank is made out of resonators, it is more useful to talk about the coupling in terms of Qs rather than capacitances as shown in the circuit diagram. Additionally, in the current SuperSpec dies the resonators are proximity coupled and the coupling includes both capacitive and inductive terms. The resonator Q can be broken up into three quantities:

$$
\frac{1}{Q}=\frac{1}{Q_{c}}+\frac{1}{Q_{i}}+\frac{1}{Q_{\text {loss }}}
$$

Here, these Qs are defined with $Q_{c}$ being the coupling Q to the feedline, $Q_{\text {loss }}$ being the intrinsic $\mathrm{Q}$ of the resonator, dominated by the loss of the dielectric, and finally $Q_{i}$ being the $\mathrm{Q}$ defining the coupling to the detector. The other relevant parameter of the filterbank is the oversampling (denoted by the symbol $\Sigma$ ). This is the number of resonators packed into a single linewidth, as defined by the individual resonator $\mathrm{Q}$. This is illustrated in fig. 2, where a simulation of a filterbank response is shown versus frequency with a filterbank having an oversampling of 1.0 on the left and 2.0 on the right.

We use a circuit model implemented in python using the Scikit-RF module (G. Che et al., submitted) to select the values of $Q_{c}, Q_{i}$, and $\Sigma$ that deliver the optimal filterbank sensitivity. Previous SuperSpec devices have found a $Q_{\text {loss }}$ around 1200-1300, and this keeps the optimal oversampling factor close to $\Sigma=2$. With $\Sigma<2$, there is not significant overlap in the passbands of the adjacent channels and so the quarter-wave spacing gives little improvement, with $\Sigma>2$, a larger filterbank is needed and the dielectric loss starts taking a larger fraction of the incident power. With $Q_{\text {loss }}=1200$ and $\Sigma=2$ the filterbank sensitivity is optimized for $Q_{c} \approx 700$ and 

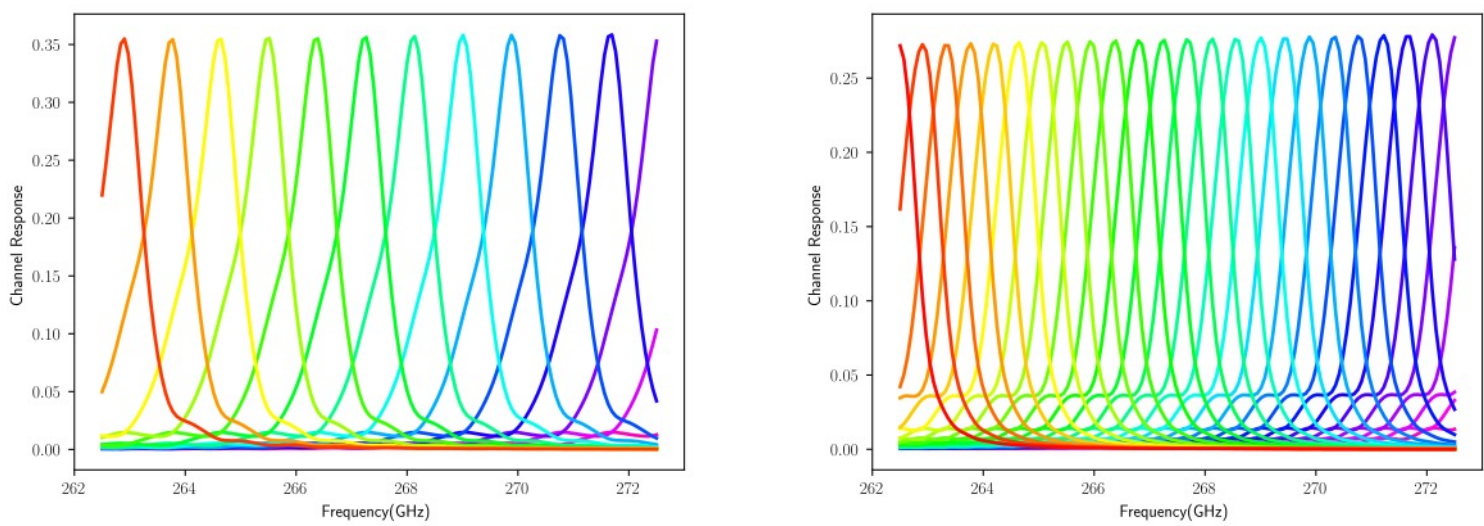

Figure 2. The simulated response of a filterbank with an oversampling value of 1.0 on the left, and 2.0 on the right. Each color is the response of a different spectral channel.

$Q_{i} \approx 700$. It is important to note that in addition to the usual consideration of the resonator being well matched so that the power couples in, there are two further considerations added by our filterbank. The first being that even when not optimally matched, the overlap of the spectral response means that much of the uncoupled power could still end up in another channel and the spectral line recovered in a deconvolution taking into consideration this affect. Second, the ratio of $Q_{\text {loss }}$ to $Q_{i}$ will set the maximum ratio of power that ends up on the detectors, as this ratio will set the relative rates of power coupled into the resonator that is absorbed by the inductor versus power lost to the dieletric. So with that in mind, the optimization ends up finding that these minima are very shallow, and variations in Q by a factor of 2 only give a 20 to 30 percent deviation in the total system NEP (fig. $3)$.

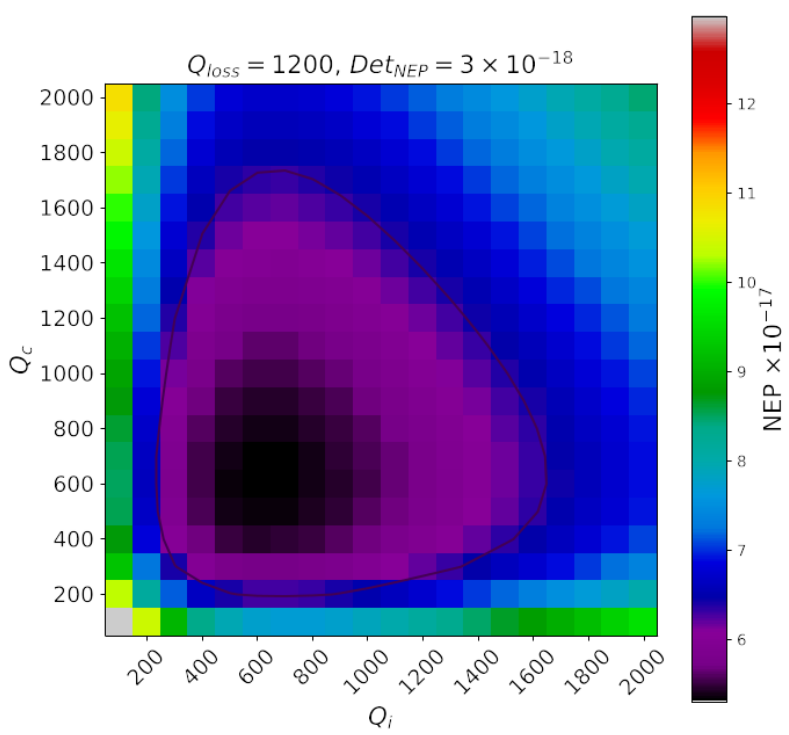

Figure 3. A plot of the total system NEP for a narrow spectral line, referenced to the input at the front of the cryostat, for various $Q_{c}$ and $Q_{i}$, given $Q_{\text {loss }}=1200$, a detector NEP of $3 \times 10^{-18} \mathrm{~W} \mathrm{~Hz}^{-1 / 2}$, and an oversampling factor of $\Sigma=2$. The contour shown highlights where the total system NEP reaches 20 percent greater than the minimal value.

SuperSpec devices are implemented using an inverted microstrip design, allowing for the ground plane and dielectric to be removed from around the capacitors of the LEKIDs, reducing TLS noise. ${ }^{9}$ The devices use both 


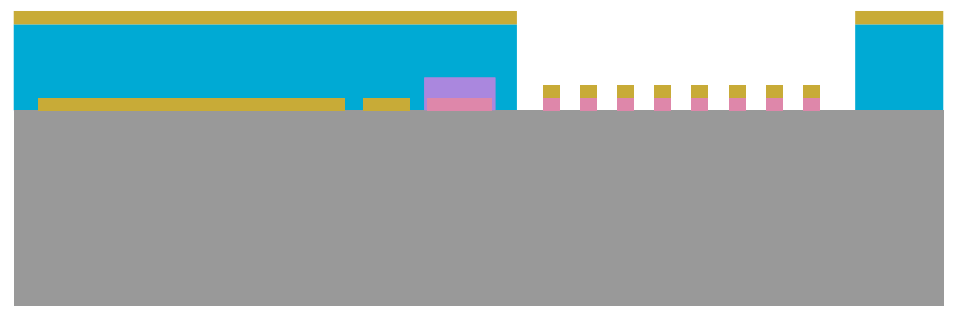

Figure 4. A diagram of the layer stack. First, the 20nm TiN layer (magenta) is deposited and patterned to define the KIDs. Next silicon oxide (purple) is deposited to protect the TiN inductors from being etched or damaged by future processing. The first niobium (gold) layer is then deposited and patterned. The $0.5 \mu \mathrm{m}$ silicon nitride dielectric (light blue) and niobium ground plane are deposited, and both are then patterned and etched in the same step, exposing the capacitors of the KIDs and the antenna slots.

niobium and titanium nitride (TiN), with the niobium being a high $T_{c}$ superconductor that will not experience pair breaking due to the $\mathrm{mm}$ radiation, and the TiN being the material of the KID inductors used for sensing. ${ }^{10}$ The device stack is shown in fig. 4. The layout of the next generation of SuperSpec devices intended for the telescope demonstration is shown in fig. 5 .

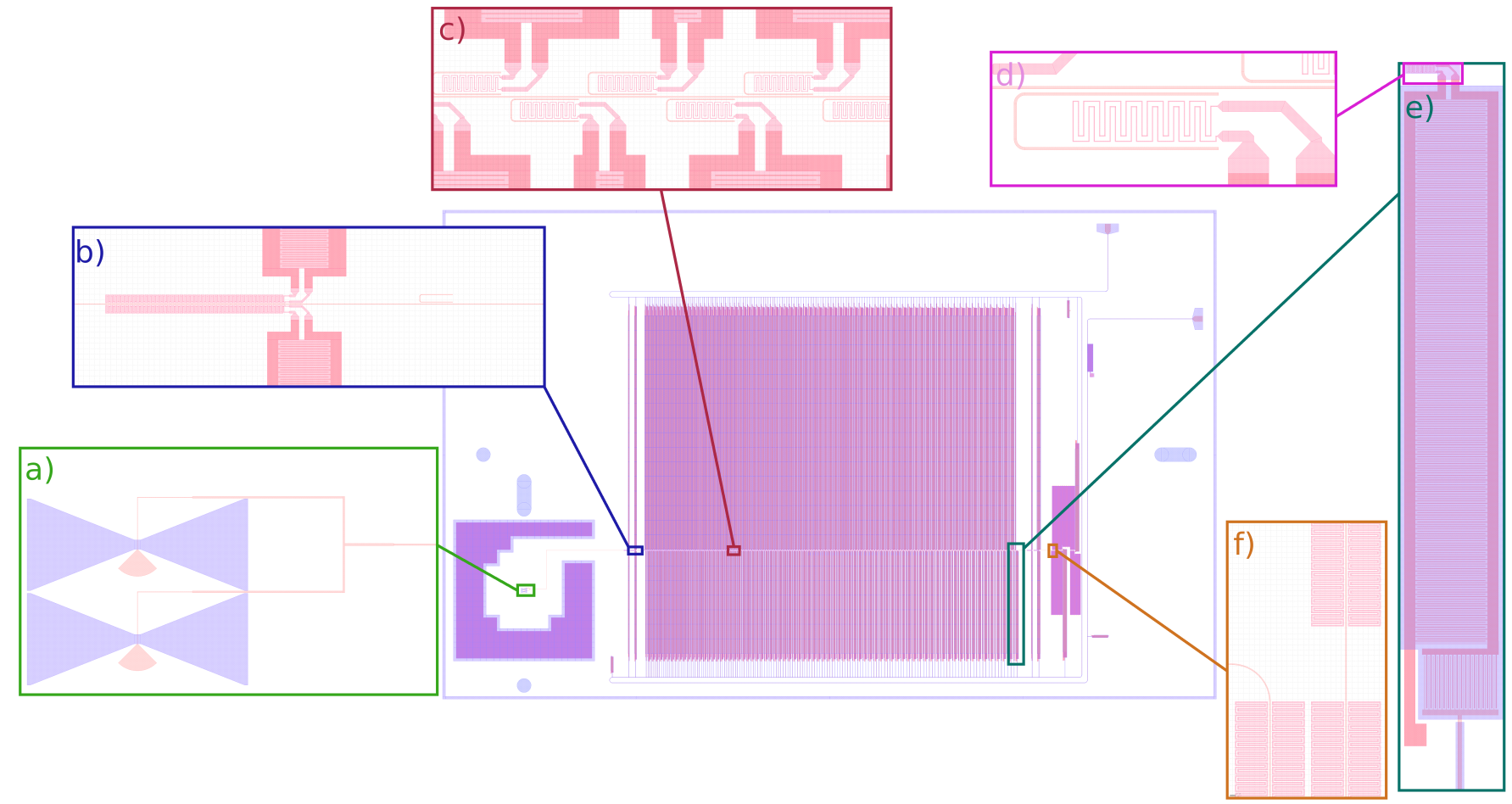

Figure 5. Shown is the layout of the 300 channel SuperSpec chip. a) The antenna that couples the radiation into the feedline. b) Test structures; on the left is a pair of broadband detectors (KIDs without half-wave resonators that are sensitive to the entire band), and on the right is a half-wave resonator with no KID, isolated in frequency, used measure the internal Q of the filterbank resonators. c) A section of the filterbank, showing several half-wave resonators wrapped around the inductors of KID detectors. d) A single spectral filter, highlighting the $2.7 \mu \mathrm{m}^{3}$ inductor, with $0.25 \mu \mathrm{m}$ traces. e) A single spectral channel and associated KID. f) The termination, which absorbs the radiation that makes it throught the filterbank.

A double "bowtie" antenna provides both the coupling over the large bandwidth, and in conjunction with a $1 \mathrm{~cm}$, anti-reflection coated alumina lens, produces a roughly $\mathrm{f} / 4$ beam over the whole band. Simulations of the design along with measured beams from our beam mapper are shown in fig. 6. After being coupled into 
the microstrip by the antenna, the radiation then travels down a $1 \mu \mathrm{m}$ microstrip to the filterbank (fig. 5 ). In addition to the spectral channels of the filterbank, there are several important test devices. First, before and after the filterbank there are a set of broadband channels. These are KIDs, the inductors of which are shown in sub-panel b) of fig. 5, that have no resonator mediating its coupling to the feedline. This causes it to detect roughly $0.1 \%$ of the power on the feedline across the entire frequency band, allowing $\mathrm{S}_{21}$ measurements of the entire filterbank and the ability to read out the loss test structures. The loss test structures are lone half-wave resonator without a KID, and one is shown in the same sub-panel. They are isolated in frequency from the filterbank, and are readout as dips in the $\mathrm{S}_{21}$ measurements. Their purpose is to provide a measurement of $Q_{\text {loss }}$. There are also "dark devices", KIDs not on the feedline, for diagnosing chip $T_{c}$ variations and radiation in substrate modes.
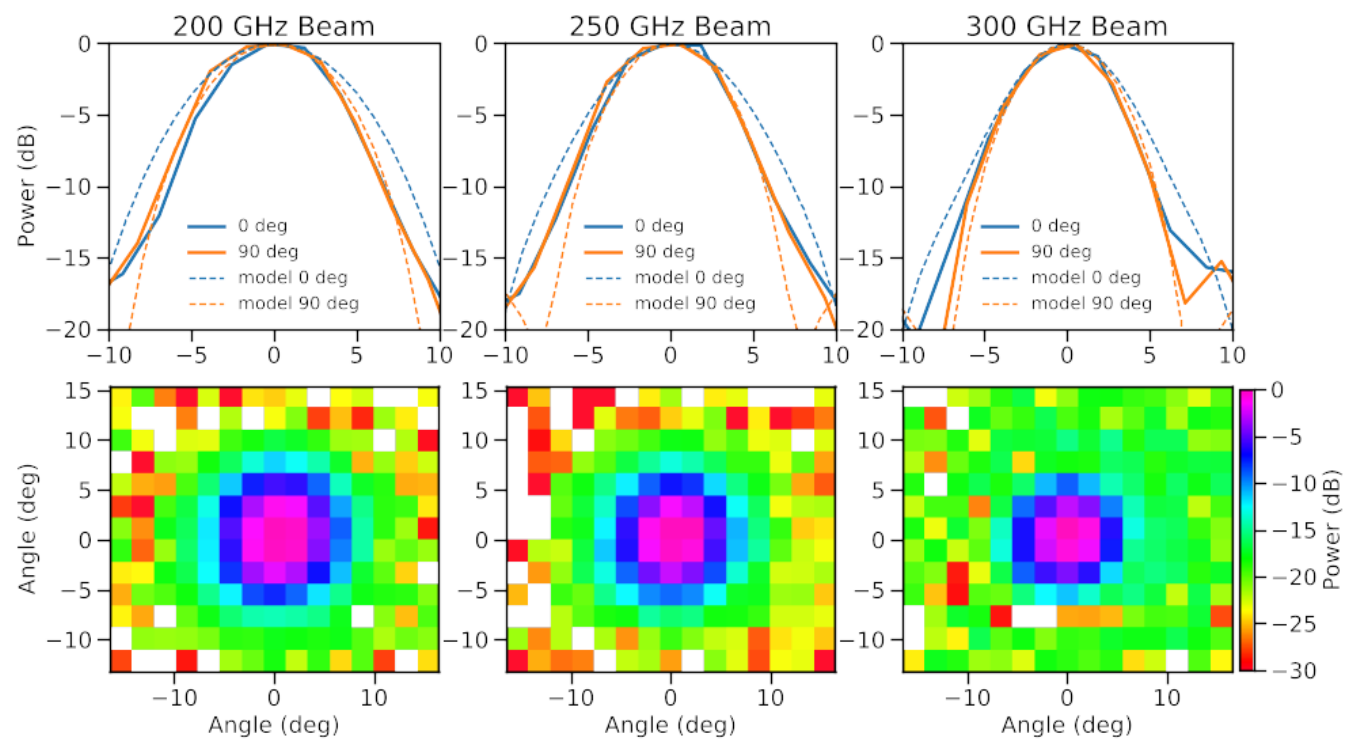

Figure 6. Shown is a plot of the simulated and measured far-field antenna beams at 200, 250, and $300 \mathrm{GHz}$. The top row is a series of 1-D cross sections of the beam with the simulated beams in dashed lines and the measured beams in solid lines. Bottom is the 2-D beam map measured 0.3 meters from the front of the cryostat. White blocks are where noise gave a negative or zero response, necessitating dropping them from a log plot.

The method of efficiently utilizing the area of the chip for capacitor layout was implemented by breaking the readout frequency into four banks. Since the resonant frequency scales as $f \propto \frac{1}{\sqrt{C}}$, each bank is set to have the frequency scale over a factor of $\sqrt{315 / 185}$ so that they scale their capacitance with the quarter wave spacing. This way, the resonators can all be set to the same length and the area is optimally utilized. Then the relative widths of each bank and the length on each side of the feedline can be set to give the necessary ratio of frequencies between the two banks (fig. 7).

\section{SUPERSPEC CHARACTERIZATION}

The beam maps shown in fig. 6 were taken using a beam mapper mounted under the cryostat window with a $0.5 \mathrm{~m} x$ and y travel, and a source made from a one inch hole into a liquid nitrogen bucket, which was chopped at $5 \mathrm{~Hz}$. Additionally, a $1 \mathrm{~m}$ throw Fourier Transform Spectrometer was used for measuring the spectral response of the filterbank, providing a measurement of the central frequency of each spectral channel. With the first 300 channel prototype we were able to demonstrate the ability to target the frequency of the individual filterbank channels over the entire band (fig. 8). A fabrication defect greatly reduced our yield for this device; we expect this issue to be solved in future fabrication runs. Previously, we have demonstrated the ability to create a 50 channel spectrometer over a subset of our band with 100 percent yield. ${ }^{11}$ We have also demonstrated in past devices a photon noise limited NEP for our detectors. ${ }^{12}$ 


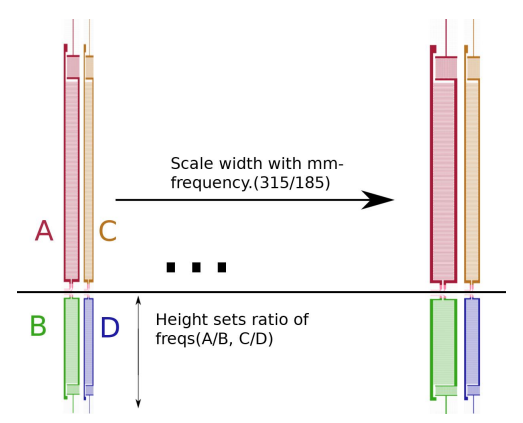

Figure 7. A diagram of the capacitor/frequency layout of the KIDs. To optimally use the area, the readout band was split into 4 so that the the capacitance scaling of each is $\sim 1.7$, equal to the quarter wave scaling of the resonators accross the filterbank. Therefore, the frequencies in the band can be set by scaling the width of the capacitors with the quarter wave spacing. The four banks are interspersed on each side of the feedbank (each band of the readout is represented here by a different color). Therefore, the four banks can have their relative ratios set by the relative widths of adjacent capacitors and the lengths of the capacitors on each side of the readout line.

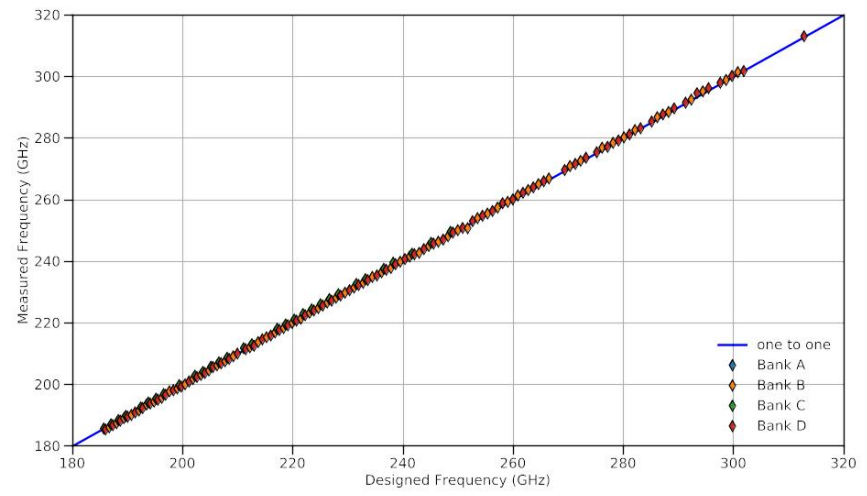

Figure 8. Shown is a plot of the designed versus measured frequency of the filterbank resonators for the first 300 channel device. This measurement demonstrates the ability to properly target the channel frequency over the entire band.

\section{DEPLOYMENT AT THE LMT}

We are designing a three beam, dual-polarization SuperSpec demonstration instrument for deployment at the LMT in early 2019. A polarizing grid near the instrument focal plane will split the incoming light, which will then be received by a total of six single-polarization spectrometer chips (fig. 9).

The SuperSpec coupling optics consist of the warm mirrors under development for MUSCAT, ${ }^{13}$ along with a subsequent set of 5 warm mirrors, and a single cold lens (fig. 9). A flat pickoff mirror placed in front of the MUSCAT cryostat diverts the beam, and a second flat mirror sends the beam toward the SuperSpec reimaging optics. This second flat mirror is located at an image of the telescope primary mirror and will be mounted on an articulated stage. Chopping this mirror will provide rapid on-sky modulation. A set of two powered mirrors and one flat mirror then reimage this pupil onto a $4 \mathrm{~K}$ cold stop. A subsequent $1 \mathrm{~K}$ lens then provides a flat, telecentric, $\mathrm{f} / 4$ focus. While the three beams in the intial SuperSpec demonstrator will span a linear 1' field on the sky, the coupling optics will be sufficiently large to reimage a 2' circular field with a Strehl ratio $>0.92$ at $\lambda=1 \mathrm{~mm}$.

The readout system will be based off of kidPy*, a product of the development of the BLAST-TNG readout system, ${ }^{14}$ with 6 ROACH-2 systems, one for each chip, to simplify readout for this demonstration. Custom interface software is under development for integrating the data on the telescope pointing, chopping mirror, and simultaneously controlling the 6 ROACH-2 systems.

\footnotetext{
*https://github.com/sbg2133/kidPy
} 

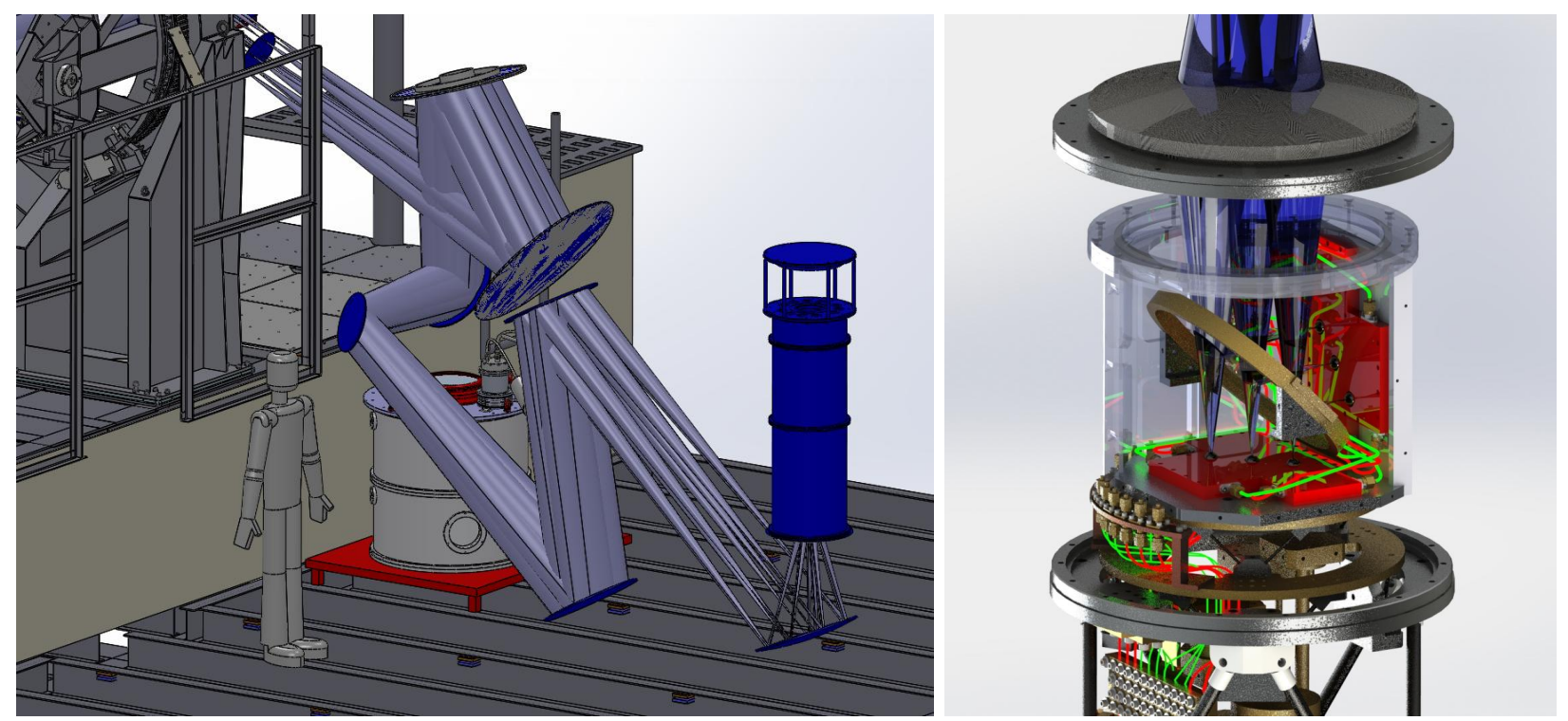

Figure 9. (left) A CAD rendering of the instrument at the LMT, highlighting the optical path for a 2' field of view. (right) A cutaway view of the cryostat showing the three beams incident on the cold lens, and being separated by a polarizing grid into 6 beams detected by 6 devices.

\section{CONCLUSION}

We have designed and fabricated an optimized 300 channel on-chip filterbank spectrometer covering the $185-315$ $\mathrm{GHz}$ range with a resolving power of $\mathcal{R} \sim 310$. Initial testing has demonstrated our ability to accurately place the spectral channels over the full band, and has also demonstrated a new readout frequency architecture. The next set of dies combining all of the design elements, with Qs further optimized, and fixing the low yield of our last device is currently being fabricated and is anticipated to be ready for deployment at the LMT.

\section{REFERENCES}

[1] Herrera-Camus, R., Bolatto, A. D., Wolfire, M. G., Smith, J. D., Croxall, K. V., Kennicutt, R. C., Calzetti, D., Helou, G., Walter, F., Leroy, A. K., Drai ne, B., Brandl, B. R., Armus, L., Sandstrom, K. M., Dale, D. A., Aniano, G., Meidt, S. E., Boquien, M., Hunt, L. K., Galametz, M., Tabatabaei, F. S., Murphy, E. J., Appleton, P., Roussel, H., Engelbracht, C., and Beirao, P., "[CII] $158 \mu \mathrm{m}$ Emission as a Star Formation Tracer," (2014).

[2] Bradford, C. M., Hailey-Dunsheath, S., Shirokoff, E., Hollister, M., McKenney, C. M., LeDuc, H. G., Reck, T., Chapman, S. C., Tikhomirov, A., Nikola, T., and Zmuidzinas, J., "X-Spec: a multi-object transmillimeter-wave spectrometer for CCAT," in [Millimeter, Submillimeter, and Far-Infrared Detectors and Instrumentation for Astronomy VII], Proceedings of the SPIE 9153, 91531Y (Aug. 2014).

[3] Serra, P., Doré, O., and Lagache, G., "Dissecting the high-z interstellar medium through intensity mapping cross-correlations," The Astrophysical Journal 833(2), 1-13 (2016).

[4] Gong, Y., Cooray, A., Silva, M., Santos, M. G., Bock, J., Bradford, C. M., and Zemcov, M., "Intensity mapping of the [CII] fine structure line during the epoch of reionization," Astrophysical Journal $\mathbf{7 4 5}(1)$ (2012).

[5] Bouwens, R. J., Illingworth, G. D., Oesch, P. A., Trenti, M., Labbe', I., Bradley, L., Carollo, M., van Dokkum, P. G., Gonzalez, V., Holwerda, B., Franx, M., Spitler, L., Smit, R., and Magee, D., "UV Luminosity Functions at redshifts z 4 to z 10: 11000 Galaxies from HST Legacy Fields," ArXiv e-prints (Mar. 2014).

[6] Bouwens, R. J., Illingworth, G. D., Oesch, P. A., Trenti, M., Labbé, I., Bradley, L., Carollo, M., van Dokkum, P. G., Gonzalez, V., Holwerda, B. a nd Franx, M., Spitler, L., Smit, R., and Magee, D., "UV Luminosity Functions at Redshifts $\mathrm{z} \sim 4$ to $\mathrm{z} \sim 10:$ 10,000 Galaxies from HST Legacy Fields," The Astrophysical Journal 803, 34 (Apr. 2015). 
[7] Pardo, J. R., Cernicharo, J., and Serabyn, E., "Atmospheric transmission at microwaves (ATM): An improved model for millimeter/submillimeter applications," IEEE Transactions on Antennas and Propagation 49(12), 1683-1694 (2001).

[8] Kov(á)cs, A., Barry, P. S., Bradford, C. M., Chattopadhyay, G., Hailey-dunsheath, S., Hollister, M., Mckenney, C., Leduc, H. G., Llombart, N., Marrone, D. P., Mauskopf, P., Brient, R. O., Padin, S., Swenson, L. J., and Zmuidzinas, J., "SuperSpec: design concept and circuit simulations,"

[9] Gao, J., Daal, M., Martinis, J. M., Vayonakis, A., Zmuidzinas, J., Sadoulet, B., Mazin, B. A., Day, P. K., Leduc, H. G., Gao, J., Daal, M., Martinis, J. M., Vayonakis, A., Zmuidzinas, J., Sadoulet, B., Mazin, B. A., Day, P. K., and Leduc, H. G., "A semiempirical model for two-level system noise in superconducting microresonators A semiempirical model for two-level system noise in superconducting microresonators," Applied Physics Letters 212504(92), 1-4 (2008).

[10] Leduc, H. G., Bumble, B., Day, P. K., Eom, B. H., Gao, J., Golwala, S., Mazin, B. A., McHugh, S., Merrill, A., Moore, D. C., Noroozian, O., Turner, A. D., and Zmuidzinas, J., "Titanium nitride films for ultrasensitive microresonator detectors," Applied Physics Letters 97(10), 2008-2011 (2010).

[11] Wheeler, J., Hailey-Dunsheath, S., Shirokoff, E., Barry, P. S., Bradford, C. M., Chapman, S., Che, G., Glenn, J., Hollister, M., Kovcs, A., LeDuc, H. G., Mauskopf, P., McGeehan, R., McKenney, C. M., OBrient, R., Padin, S., Reck, T., Ross, C., Shiu, C., Tucker, C. E., Williamson, R., and Zmuidzinas, J., "Superspec: development towards a full-scale filter bank," Proc.SPIE 9914, $9914-9914-9$ (2016).

[12] Wheeler, J., Hailey-Dunsheath, S., Shirokoff, E., Barry, P. S., Bradford, C. M., Chapman, S., Che, G., Doyle, S., Glenn, J., Gordon, S., Hollister, M., Kovács, A., LeDuc, H. G., Mauskopf, P., McGeehan, R., McKenney, C., Reck, T., Redford, J., Ross, C., Shiu, C., Tucker, C., Turner, J., Walker, S., and Zmuidzinas, J., "SuperSpec, The On-Chip Spectrometer: Improved NEP and Antenna Performance," Journal of Low Temperature Physics (May 2018).

[13] Castillo-Dominguez, E., Ade, P., Barry, P., Brien, T., Doyle, S., Ferrusca, D., Gomez-Rivera, V., Hargrave, P., Hornsby, A., Hughes, D., Mauskopf, P., Moseley, P., Pascale, E., Perez-Fajardo, A., Pisano, G., Rowe, S., Tucker, C., and Velazquez, M., "Mexico-UK Sub-millimeter Camera for AsTronomy," ArXiv e-prints (June 2018).

[14] Gordon, S., Dober, B., Sinclair, A., Rowe, S., Bryan, S., Mauskopf, P., Austermann, J., Devlin, M., Dicker, S., Gao, J., Hilton, G. C., Hubmayr, J., Jones, G., Klein, J., Lourie, N. P., McKenney, C., Nati, F., Soler, J. D., Strader, M., and Vissers, M., "An open source, fpga-based lekid readout for blast-tng: Pre-flight results," Journal of Astronomical Instrumentation 05(04), 1641003 (2016). 